\title{
A (Critical) Review of Electroweak Symmetry Breaking
}

\author{
Csaba Csáki \\ Institute of High Energy Phenomenology \\ Laboratory of Elementary Particle Physics \\ Cornell University \\ Ithaca, NY 14853, USA \\ E-mail: csaki@cornell.edu
}

\begin{abstract}
An overview of models of electroweak symmetry breaking is presented. First we explain the little hierarchy problem and show how it is manifested in supersymmetric theories. Then ways of avoiding the little hierarchy in SUSY models is shown, which fall into two classes: hiding the higgs at LEP or increasing the quartic self coupling of the higgs, both of which call for extensions of the MSSM. In the second half strongly interacting theories of electroweak symmetry breaking are reviewed, including technicolor and monopole condensation models. Particular attention is paid to warped extra dimensional theories and its cousins (higgsless, little higgs and composite higgs models.
\end{abstract}

35th International Conference of High Energy Physics - ICHEP2010,

July 22-28, 2010

Paris France 


\section{The SM, big vs. little hierarchy}

The standard Higgs mechanism is an eminently successful description of electroweak symmetry breaking. The analysis of electroweak precision data suggest that there is a light weakly coupled higgs boson, below about $200 \mathrm{GeV}$. However, it is very hard to understand how such an elementary higgs would remain light. Examining quantum corrections one finds that the higgs mass is quadratically sensitive to any new physics:

$$
\Delta m_{H}^{2} \sim \frac{g^{2}}{16 \pi^{2}} \Lambda^{2}
$$

where $\Lambda$ is the scale of new physics. One should emphasize that this sensitivity could just be a finite contribution from a new physics coupling to the higgs boson, so it does not necessarily imply the presence of any divergences. This is what is usually referred to as the big hierarchy problem: why is $m_{H} \ll \Lambda$. The most straightforward resolution of this problem would be that the scale of new physics is actually low, $\Lambda \sim 1 \mathrm{TeV}$, and whatever that new physics is it will make the higgs insensitive to further quantum corrections above the $\mathrm{TeV}$ scale (for example via the appearance of superpartners, or of strong dynamics). The little hierarchy refers to the puzzle of why have we not yet seen some indirect hints for the existence of any of these new particles that are supposed to be showing up at $1 \mathrm{TeV}$. In fact in most models electroweak precision observables force the scale of new physics to be more like 5-10 $\mathrm{TeV}$, leading to a new fine tuning of order of $\sim 1$ percent. Supersymmetric theories are somewhat special, since in that case R-parity protects electroweak precision observables from tree-level corrections, which would imply $4 \pi m_{S U S Y} \sim 5-10 \mathrm{TeV}$, thus superpartners of $500 \mathrm{GeV}-\mathrm{TeV}$ could still be allowed. However, as we will show below the minimal supersymmetric extension of the standard model (MSSM) is nevertheless plagued by a little hierarchy problem, somewhat specific to supersymmetry.

\section{The little hierarchy in the MSSM}

The little hierarchy problem of the MSSM is mainly due to the fact that the Higgs quartic self interaction term is related to the gauge couplings by supersymmetry. This quartic is essential since it sets the size of the physical higgs boson mass (like in the SM $m_{H}=\sqrt{2 \lambda} v$ where $\lambda$ is the quartic). In the MSSM there are two higgs doublets $H_{u, d}$, both of which have to obtain a VEV to yield fermion and gauge boson masses. Only one combination of these receives a quartic (the direction along which the two VEVs are equal will not have a quartic). By making one VEV much bigger than the other (the so called large $\tan \beta$ limit) the quartic can be maximized, and in this limit one finds that the lightest higgs boson mass at one loop is

$$
m_{H}^{2}=M_{Z}^{2}+\frac{3 m_{t}^{2} \lambda_{t}^{2}}{4 \pi^{2}} \log \frac{m_{\tilde{t}}}{m_{t}}
$$

where $m_{t, \tilde{t}}$ are the top and stop masses, and $\lambda_{t}$ is the top yukawa coupling. In order to push this above $114 \mathrm{GeV}$ (the bound at LEP2) one needs very large one-loop corrections: in fact the one loop corrections have to be almost as large as the tree-level term. However supersymmetry is introduced in order to soften the one-loop contributions! Here one needs to secretly reintroduce a not-so-small 
loop correction, and that can only be done by increasing the top-stop splitting. However the same splitting contributes to the soft breaking mass parameter of the higgs boson:

$$
m_{H_{u}}^{2}=m_{0}^{2}-\frac{3 \lambda_{t}^{2} m_{\tilde{t}}^{2}}{4 \pi^{2}} \log \frac{\Lambda^{2}}{m_{\tilde{t}}^{2}}
$$

This parameter however gives a direct contribution to the Z-mass and is expected $M_{Z} \sim m_{H_{u}}$, resulting in the generic $0.1-1 \%$ tuning of the MSSM.

Clearly, this little hierarchy problem is related to the size of the Higgs quartic. There are two distinct potential ways to get around this problem: either the quartic is indeed small, but the higgs eluded detection at LEP since it has unconventional decays, or there need to be additional contributions to the quartic. Both call for extensions of the MSSM.

\subsection{Hiding the Higgs at LEP}

An interesting possibility recently explored by many groups $[1,2]$ is that the Higgs has unconventional decays to a pseudo-scalar $\eta$ with the dominant higgs decay $h \rightarrow 2 \eta$, with the $\eta$ later decaying to two SM particles. The most popular possibility for a long while has been the decay $h \rightarrow 2 \eta \rightarrow 4 \tau$. However a recent re-analysis [3] of the ALEPH data showed that this channel was almost as strongly constrained as the SM channels. The LEP constraints as of the time of this conference are summarized in Table 1 . One can see that one possibly promising way to hide the higgs is by having it decay to 4 light jets via an intermediate state with two pseudo-scalars. This can be achieved both within the NMSSM, and via an extension of the MSSM assuming an SU(3) global symmetry [4]. In this case the higgs as well as an additional pseudo-scalar $\eta$ form the 5 Goldstone bosons from the breaking $\mathrm{SU}(3) \rightarrow \mathrm{SU}(2)$, and the requisite $h \eta \eta$ coupling shows up as a derivative coupling between the Goldstones. If the global symmetry breaking scale $f$ is sufficiently small $f \sim 350-400 \mathrm{GeV}$, this can indeed be the dominant decay channel. The actual final state depends on the further decay of the $\eta$, which in turn depends on the embedding of the SM fermions into the SU(3) global symmetry. The most natural forms (where the global SU(3) is a remnant of a full gauge SU(3) extension of the electroweak) are the cases when the extended gauge anomalies all vanish. In this case one can show that the $\eta$ will preferentially decay either to gluons [4] (which was dubbed "the buried higgs") or to charm quarks [5] (which is referred to as the "charming higgs"). In both cases the final states are four non-b jets, which will be quite hard to observe at the LHC. Methods for finding these buried higgs like scenarios using the methods of jet substructure were given in [6].

\subsection{Other SUSY approaches}

The other option of solving the little hierarchy problem of the MSSM is to find a way to increase the quartic self-coupling of the Higgs without introducing additional quadratic divergences to the higgs bosons. Here I present a list of the options that have been considered recently.

- An NMSSM quartic [7]. Introducing the singlet $S$ the $\mu$-term can be replaced by $\lambda S H_{u} H_{d}$ in the superpotential. Raising $\lambda$ will increase the quartic, though a bound of $m_{h}<150 \mathrm{GeV}$ still applies if one requires that a Landau pole for $\lambda$ is pushed above the GUT scale. 


\begin{tabular}{|l|c|}
\hline Decay channel & Limit $(\mathrm{GeV})$ \\
\hline$h \rightarrow b \bar{b}, \tau \bar{\tau}$ & 115 \\
$h \rightarrow j j$ & 113 \\
$h \rightarrow \gamma \gamma$ & 117 \\
$h \rightarrow W W^{*}, Z Z^{*}$ & 110 \\
$h \rightarrow$ invisible & 115 \\
\hline$h \rightarrow \eta \eta \rightarrow 4 b, 4 \tau$ & 110 \\
$h \rightarrow \eta \eta \rightarrow 4 c, 4 g$ & 86 \\
model indep. & 82 \\
\hline
\end{tabular}

Table 1: The LEP bounds on the mass of the Higgs boson in various decay channels as of July 2010.

- A "fat higgs" [8]. In this case the NMSSM Landau-pole is replaced by a weakly coupled Seiberg dual once the interaction becomes strong. The higgs is effectively a composite object, and the large quartic the effect of the underlying strong dynamics.

- A non-renormalizable quartic superpotential [9]. An NMSSM-like effective theory can be obtained without the introduction of a singlet, but from a $\left(H_{u} H_{d}\right)^{2}$-like non-renormalizable term in the superpotential.

- A non-decoupling D-term [10]. Usually D-terms from additional gauge interactions decouple if the gauge symmetry breaking is completely supersymmetric. However if $m_{s o f t} \sim$ the VEV of the field breaking the gauge symmetry then the D-terms may not fully decouple, and might raise the higgs mass to as high as $400 \mathrm{GeV}$.

\section{Models of strong dynamics}

\subsection{Technicolor type theories}

It is well-known that an elementary higgs boson may not be necessary for electroweak symmetry breaking. The vacuum structure of a strongly interacting gauge theory might break the electroweak symmetry just as it does in QCD: here the quarks form vacuum condensates $\left\langle u_{L} u_{R}\right\rangle=$ $\left\langle d_{L} d_{R}\right\rangle \sim f_{\pi}^{3}$. These condensates do give rise to masses for the $\mathrm{W}$ and the $\mathrm{Z}$ boson, except their contributions are too small by about a factor of $10^{3}$. The most simple remedy for this is to introduce a scaled-up QCD called technicolor, with $\Lambda_{T C} \sim \mathrm{TeV}$. This will give the right gauge boson masses, however it faces two difficult problems:

- Electroweak precision corrections. The $S$-parameter in generic technicolor theories is usually assumed to be too large. If a scaled-up QCD-like contribution was reliable the resulting $S$ parameter would be of the order $S \sim 0.28 N_{D} \frac{N_{T C}}{3}$, where $N_{D}$ is the number of additional $\mathrm{SU}(2)$ doublets introduced that participate in the strong technicolor interactions, and $N_{T C}$ is the number of technicolors. However the $S$-parameter is generically not calculable, and thus it is not clear whether or not some non-QCD-like technicolor theories could produce a small $S$-parameter. 
- Fermion masses. There is a built-in tension in generic technicolor models when one considers fermion masses. The SM quark masses should be produced by 4-fermi operators of the sort $\frac{1}{\Lambda_{F}^{2}} \bar{q} q \bar{\psi} \psi$ where $q$ are SM fermions and $\psi$ are technifermions. In order to obtain a sufficiently heavy top mass the scale $\Lambda_{F}$ should not be too large $<10 \mathrm{TeV}$. However the same physics will generically also give rise to flavor changing four SM fermion operators of the form $\frac{1}{\Lambda_{F}^{2}} \bar{q} q \bar{q} q$. Experiments require that this scale be quite large $>10^{4} \mathrm{TeV}$. Possible ways to get around this problem involve walking technicolor, where a large anomalous dimension of $\bar{\psi} \psi$ relives some of the tension, and conformal technicolor [11] where the dimension of the composite $\bar{\psi} \psi$ is close to one, thereby acting almost like an elementary higgs from the point of view of flavor physics. However recent results on the simplest conformal field theories presented at this conference [12] suggest that it is difficult to sufficiently suppress the FCNC's without the hierarchy reemerging.

\subsection{Monopole condensation}

A recent new idea [13] for replacing technicolor theories is based on magnetic monopoles. The Dirac quantization condition $e g=2 \pi n$ implies that if the electric coupling $e$ is small, then the magnetic coupling $g$ is necessarily large. Thus if there were magnetically charged chiral fermions (and assuming that the running of the $\mathrm{U}(1)$ gauge coupling is still driven by the electric degrees of freedom) the magnetically charged particles would necessarily condense thus resulting in a similar dynamically broken phase for the electroweak interactions as in technicolor models. A toy model that might potentially be in this phase is based on an extension of the SM with a fourth generation of chiral fermions, which however also carry magnetic $\mathrm{U}(1)_{Y}$ charges proportional to their $B-L$ quantum numbers. This is an anomaly free theory [14], and can incorporate the right monopole condensates that can act like the ordinary higgs VEV. A small modification of the model in analogy with ordinary GUT-like monopole charge assignments can also ensure that the condensates do not confine the ordinary electric photon and that magnetic couplings only affect the photon, but not the $\mathrm{W}$ and the Z. This way one would find a theory with massive QED-like monopoles above the scale of condensation, which could be pair-produced at the LHC. The main drawback of this model is that due to the Dirac quantization condition the theory is always strongly interacting, there is no regime where perturbation theory is well defined. Thus it is hard to make any quantitative predictions for LHC cross sections (or even to verify that the right condensates actually do form).

\subsection{Warped extra dimensions}

A very popular theme of the past decade has been the study of warped extra dimensions as models of electroweak symmetry breaking [15]. In such theories a single extra dimension with the metric

$$
d s^{2}=\left(\frac{R}{z}\right)^{2}\left(d x^{2}-d z^{2}\right)
$$

is assumed. The reason why such theories are so interesting for electroweak phenomenology is that the mass scales vary along the extra dimension the same way as the metric factor. If one assumes that the extra dimension is a finite slice of the anti-de Sitter space, with boundaries at $z=R$ ("UV brane" or "Planck brane") and $z=R^{\prime}$ ("IR brane" or "TeV brane"), then all physical quantities will 
be rescaled by the warp factor $R / R^{\prime}$. In particular, a Higgs field localized on the IR brane will have a physical value of $v_{\text {eff }}=v_{\text {bare }} R / R^{\prime}$. This implies that picking all bare quantities of the order of the Planck scale $1 / R \sim v_{\text {bare }} \sim M_{P l}$ and $1 / R^{\prime} \sim \mathrm{TeV}$ one can naturally recover the exponential hierarchy between the weak and the Planck scales [15].

A very nice interpretation of this solution to the hierarchy problem can be obtained in terms of the AdS/CFT duality [16]: based on this such 5D models with a warped space can be interpreted in terms of a strongly interacting technicolor-like theory. The fifth dimension can be viewed as the energy scale of a 4D field theory, and the emergence of the IR brane is corresponding to confinement and dynamical symmetry breaking. Thus all fields that are localized on the IR brane correspond to composites of the strong dynamics, and thus can be naturally light (the mass scale for the is set by the strong dynamics scale), while the field localized close to the UV brane are elementary, and their natural mass scale is very large.

The original Randall-Sundrum model was assuming that all the SM fields are localized on the IR brane. Thus the solution of the hierarchy problem in this case would be that all SM fields are composites of a strong dynamics. While this is possible, there are good reasons to think that this might not be the ideal setup: if fermions and gauge bosons are composite, then it is very hard to understand why there would be no large flavor changing neutral currents (FCNC's) induced for the SM fermions on the IR brane, and similarly large contributions to the gauge boson Lagrangian inducing corrections to electroweak precision observables. However, in order to solve the hierarchy problem one really only needs the higgs to be on the IR brane! Thus a more realistic version of warped extra dimensional models has emerged: the higgs is localized on the IR brane, the gauge fields are in the bulk [17] (and their lightest KK modes corresponding to the SM gauge fields mostly flat), while the SM fermions are in the bulk but [18] localized close to the UV brane, signifying the fact that these are mostly elementary fields. The lightness of the SM fermions is then explained via the fact that these fields are localized far from the source of electroweak symmetry breaking, and thus can not pick up a large mass (in the AdS/CFT language the SM fermions are mixtures of elementary and composite states, and there is only a very small mixing). The only exception is the top quark, which is quite heavy, so it should be more of a composite mode then elementary. In order to have additional protection against electroweak precision corrections the bulk gauge group can be extended to $\mathrm{SU}(2)_{L} \times \mathrm{SU}(2)_{R} \times \mathrm{U}(1)_{B-L}$ to incorporate custodial symmetry and thus set the $T$-parameter to zero [19]. A contribution to the $S$-paremeter of size $S \sim 12 \pi v^{2} / m_{K K}^{2}$ will remain, which will give a lower bound on the KK mass scale of $m_{K K}>3 \mathrm{TeV}$. The main observable at the LHC would be the lightest KK-mode of the gluon, which (since it is peaked towards the IR brane) is most strongly coupled to the top quark. Thus the LHC signal would be a resonance in the top production cross section, however due to the heavy mass the top quark jets will be strongly collimated [20].

This modified RS model can give a realistic theory of electroweak symmetry breaking, however it has its own little hierarchy problem which is left unaddressed: the cutoff scale at the IR brane is usually of the order of $10-100 \mathrm{TeV}$, and the natural size of the higgs mass loop corrections would be of order $\Delta m_{h} \sim \Lambda /(4 \pi)>1 \mathrm{TeV}$, which still corresponds to a tuning of the percent level. There are two directions for resolving this little hierarchy problem: one can let the higgs VEV be very large on the IR brane (leading to higgsless models) or one can introduce an additional global symmetry to protect the higgs from the leading loop corrections via the Goldstone theorem. 


\subsection{Higgsless models}

These are modifications of the warped models, where the higgs VEV on the IR brane is allowed to be arbitrary large [21]. In a 4D theory this would imply a very large gauge boson mass as well, which would be unacceptable. In 5D however if the higgs is localized something else happens: the gauge boson wave function is simply repelled from the IR brane and the gauge boson mass will be set by the geometry. The typical expression is $M_{W}^{2}=1 /\left(R^{\prime 2} \log R^{\prime} / R\right)$. The localized higgs boson will be very heavy and simply decouples from the SM: the theory is effectively higgsless. The unitarization [22] of the WW (and WZ) scattering amplitude happens via the exchange of the KK modes $Z^{\prime}, \gamma^{\prime}$ and $W^{\prime}$. Thus the theory has a sharp prediction: these KK modes should be all below 1 $\mathrm{TeV}$, and there are also some sum rules involving the masses and the couplings of these states that have to be satisfied, for example $g_{W W W W}=g_{W W \gamma}^{2}+g_{W W Z}^{2}+\sum_{i} g_{W W Z^{i}}^{2}$. The main LHC signal would be to search for a resonance in the WZ scattering amplitude, that can be reconstructed from final states with 3 leptons and missing energy [23]. The main drawback of this model are electroweak precision observables [24]. In the AdS/CFT language this is simply a calculable dual of technicolor models, and the $S$-parameter does turn out to be too large. However one can show that this can be canceled [25] by an appropriate adjustment in the fermion wave functions - but again at the price of a percent level tuning in the fermion sector of the theory.

\subsection{Composite pseudo-Goldstone Higgs}

The other approach to the little hierarchy problem in RS is to also make the higgs a pseudoGoldstone boson [26]. This will ensure that the higgs mass correction is of the form $\Delta m_{h}^{2} \sim$ $\left(g^{2} f^{2}\right) /\left(16 \pi^{2}\right)$ and is not set by the cutoff scale but rather by the global symmetry breaking scale $f$. This resolves could resolve the little hierarchy problem, however in the simplest case the entire higgs potential is loop generated, and one expects the quartic to also be loop suppressed $\lambda \sim g^{2} /\left(16 \pi^{2}\right)$. Thus one would expect $v / f \sim \mathscr{O}(1)$. In order to avoid this one needs usually again some amount of tuning to obtain an $\mathscr{O}(10)$ hierarchy in $v / f$.

\subsection{Little Higgs}

In little higgs models the higgs is also pseudo-Goldstone boson, but the main difference from the previous extra dimensional examples is that not the entire higgs potential is loop generated [27]. Due to the collective breaking mechanism a tree-level $\lambda \sim g^{2}$ quartic is allowed, generating all hierarchies naturally. The simplest models however have issues with electroweak precision observables [28], while the more sophisticated models [29] ("little higgs with T-parity") gets quite complicated once a full realistic theory [30] is written down.

\section{References}

[1] R. Dermisek, J. F. Gunion, Phys. Rev. Lett. 95, 041801 (2005).

[2] S. Chang, P. J. Fox, N. Weiner, JHEP 0608, 068 (2006).

[3] S. Schael et al. [ ALEPH Collaboration ], JHEP 1005, 049 (2010).

[4] B. Bellazzini, C. Csáki, A. Falkowski, A. Weiler, Phys. Rev. D80, 075008 (2009). 
[5] B. Bellazzini, C. Csáki, A. Falkowski, A. Weiler, Phys. Rev. D81, 075017 (2010).

[6] A. Falkowski, D. Krohn, L. -T. Wang et al., [arXiv:1006.1650 [hep-ph]]; C. -R. Chen, M. M. Nojiri, W. Sreethawong, JHEP 1011, 012 (2010); B. Bellazzini, C. Csáki, J. Hubisz and J. Shao, [arXiv:1012.1316 [hep-ph]].

[7] H. P. Nilles, M. Srednicki and D. Wyler, Phys. Lett. B 120, 346 (1983); J. M. Frere, D. R. T. Jones and S. Raby, Nucl. Phys. B 222, 11 (1983).

[8] R. Harnik, G. D. Kribs, D. T. Larson et al., Phys. Rev. D70, 015002 (2004).

[9] M. Dine, N. Seiberg, S. Thomas, Phys. Rev. D76, 095004 (2007).

[10] P. Batra, A. Delgado, D. E. Kaplan et al., JHEP 0402, 043 (2004).

[11] M. A. Luty, T. Okui, JHEP 0609, 070 (2006).

[12] R. Rattazzi, V. S. Rychkov, E. Tonni et al., JHEP 0812, 031 (2008).

[13] C. Csáki, Y. Shirman, J. Terning, Phys. Rev. Lett. 106, 041802 (2011).

[14] C. Csáki, Y. Shirman, J. Terning, Phys. Rev. D81, 125028 (2010).

[15] L. Randall and R. Sundrum, Phys. Rev. Lett. 83, 3370 (1999).

[16] J. M. Maldacena, Adv. Theor. Math. Phys. 2, 231 (1998); N. Arkani-Hamed, M. Porrati and L. Randall, JHEP 0108, 017 (2001); R. Rattazzi and A. Zaffaroni, JHEP 0104, 021 (2001).

[17] A. Pomarol, Phys. Lett. B 486, 153 (2000); H. Davoudiasl, J. L. Hewett and T. G. Rizzo, Phys. Lett. B 473, 43 (2000).

[18] Y. Grossman and M. Neubert, Phys. Lett. B 474, 361 (2000) [arXiv:hep-ph/9912408]; T. Gherghetta and A. Pomarol, Nucl. Phys. B 586, 141 (2000) [arXiv:hep-ph/0003129].

[19] K. Agashe, A. Delgado, M. J. May and R. Sundrum, JHEP 0308, 050 (2003).

[20] K. Agashe, A. Belyaev, T. Krupovnickas, G. Perez and J. Virzi, Phys. Rev. D 77, 015003 (2008);

B. Lillie, L. Randall and L. T. Wang, JHEP 0709, 074 (2007).

[21] C. Csáki, C. Grojean, L. Pilo et al., Phys. Rev. Lett. 92, 101802 (2004).

[22] C. Csáki, C. Grojean, H. Murayama et al., Phys. Rev. D69, 055006 (2004).

[23] A. Birkedal, K. Matchev and M. Perelstein, Phys. Rev. Lett. 94, 191803 (2005); C. Englert, B. Jager and D. Zeppenfeld, JHEP 0903, 060 (2009); A. Martin and V. Sanz, JHEP 1001, 075 (2010).

[24] G. Cacciapaglia, C. Csáki, C. Grojean et al., Phys. Rev. D70, 075014 (2004).

[25] G. Cacciapaglia, C. Csáki, C. Grojean et al., Phys. Rev. D71, 035015 (2005); R. Foadi, S. Gopalakrishna and C. Schmidt, Phys. Lett. B 606, 157 (2005).

[26] K. Agashe, R. Contino and A. Pomarol, Nucl. Phys. B 719, 165 (2005).

[27] N. Arkani-Hamed, A. G. Cohen, E. Katz and A. E. Nelson, JHEP 0207, 034 (2002).

[28] C. Csáki, J. Hubisz, G. D. Kribs, P. Meade and J. Terning, Phys. Rev. D 67, 115002 (2003); Phys. Rev. D 68, 035009 (2003); J. L. Hewett, F. J. Petriello and T. G. Rizzo, JHEP 0310, 062 (2003).

[29] H. C. Cheng and I. Low, JHEP 0309, 051 (2003).

[30] C. Csáki, J. Heinonen, M. Perelstein et al., Phys. Rev. D79, 035014 (2009). 